\title{
Memória em idoso: relação entre percepção subjetiva e desempenho em testes objetivos
}

\author{
Memory in older adults: Relationship between \\ subjective perception of memory and \\ performance in objective tests
}

\author{
Fábio Rodrigo BOURSCHEID ${ }^{1}$ \\ Luíza MOTHES \\ Tatiana Quarti IRIGARAY ${ }^{2}$
}

\begin{abstract}
Resumo
A relação entre a percepção subjetiva de memória e o desempenho em testes objetivos tem sido alvo de diversos estudos em vista de sua utilidade no diagnóstico do declínio cognitivo. Dada a ausência de consenso em relação ao tema, avaliou-se o desempenho de idosos em testes objetivos de memória, correlacionando-o com a percepção subjetiva dos participantes acerca de sua memória. Cento e cinquenta e dois idosos preencheram uma ficha de dados sociodemográficos, na qual foi incluída uma questão para avaliar a percepção subjetiva acerca de sua memória. Dados objetivos foram coletados a partir do Mini-Exame do Estado Mental, dos subtestes de Memória do Instrumento de Avaliação Neuropsicológica Breve, da Escala de Depressão Geriátrica e do Inventário Beck de Ansiedade. Correlações parciais significativas foram verificadas nas tarefas de memória de trabalho, memória episódica verbal e memória semântica de longo prazo, fornecendo evidências acerca da utilidade clínica da medida de percepção subjetiva de memória.
\end{abstract}

Palavras-chave: Idosos; Memória; Testes neuropsicológicos.

\begin{abstract}
The relationship between subjective perception of memory and performance in objective tests has been widely investigated given its clinical relevance in the diagnosis of cognitive impairment. Considering the lack of consensus about this topic, this study investigated the performance of older adults on objective memory tests and its correlation with their subjective perception of their own memory. One hundred and fifty-two elderly volunteers participated in this study and answered a sociodemographic questionnaire, which included a question to evaluate the subjective

$\boldsymbol{\nabla} \nabla \boldsymbol{\nabla}$

1 Universidade de Lisboa, Faculdade de Psicologia, Programa de Doutoramento em Psicologia Cognitiva. Lisboa, Portugal.

2 Pontifícia Universidade Católica do Rio Grande do Sul, Faculdade de Psicologia, Programa de Pós-Graduação em Psicologia. Av. Ipiranga, 6681, Prédio 11, Sala 939, 90619-900, Porto Alegre, RS, Brasil. Correspondência para/Correspondence to: T.Q. IRIGARAY. E-mail: <tatiana.irigaray@superig.com.br>.
\end{abstract}


perception of their own memory. Objective data were collected using the Mini Mental State Examination, the Geriatric Depression Scale, the Beck Anxiety Inventory, and memory subtests of the Neupsilin Brief Neuropsychological Assessment Instrument. Significant partial correlations were found in tasks related to working memory, verbal episodic memory, and long-term semantic memory providing additional evidence of the clinical relevance of the subjective perception of memory evaluation.

Keywords: Aged; Memory; Neuropsychological tests.

A percepção que idosos têm acerca de sua memória tem sido apresentada como um possível preditor do declínio cognitivo, particularmente no que se refere à própria memória (Jonker, Geerlings, \& Schmand, 2000). Esse tema ganha importância a partir da observação de que, na prática clínica, profissionais frequentemente se deparam com pacientes que relatam algum tipo de perda na qualidade e na acurácia de sua memória (Chaves et al., 2011).

A importância da discussão sobre o papel da percepção individual de memória (ou da metamemória) em idosos reflete-se na extensa literatura sobre o assunto. Exemplo disso é o que tem sido denominado clinicamente como "queixa subjetiva de memória", que representa a percepção subjetiva que indivíduos têm acerca de sua própria capacidade de armazenar e de recordar informações (Dufouil, Fuhrer, \& Alpérovitc, 2005). De fato, essa função pode estar intacta e mesmo prever com eficiência o declínio da memória em idosos (Souchay, 2007).

Nesse sentido, diversos estudos têm buscado avaliar o grau em que tais queixas podem predizer o desempenho em testes ou pelo menos sugerir a presença de algum declínio cognitivo (Hänninen et al., 1994; Jonker, Launer, Hooijer, \& Lindeboom, 1996). Por exemplo, Mattos et al. (2003) coletaram dados objetivos em uma amostra de 71 idosos através do Rey Auditory-Verbal Learning Test (RAVLT, Teste de Aprendizado Auditivo-Verbal de Rey), além de dados subjetivos obtidos por meio de uma pergunta ("Você tem tido dificuldades de memória que perturbam a sua rotina?"). Os autores demonstraram que participantes com pior desempenho em tarefas cognitivas também relataram maior número de queixas acerca de sua memória.

Um resultado similar foi obtido no estudo 152 de Cook e Marsiske (2006), a partir de uma amostra de 73 idosos. Os autores utilizaram medidas para diversos testes neuropsicológicos em diferentes domínios cognitivos, além de terem obtido medidas relacionadas à crença dos participantes acerca de sua memória através de dois instrumentos específicos: o Questionário de Funcionamento da Memória (Gilewski, Zelinski, \& Schaie, 1990) e o Questionário de Metamemória (Dixon, Hultsch, \& Hertzog, 1988). Os resultados demonstraram correlações significativas entre a percepção subjetiva e a performance na tarefa verbal de memória em idosos. Em comparação com idosos saudáveis, participantes pertencentes ao grupo com diagnóstico de comprometimento cognitivo leve apresentaram escores menores na avaliação subjetiva, ou seja, reportaram em média uma memória pior. As conclusões desse estudo indicam que as queixas subjetivas podem ser indicadores da performance cognitiva, pelo menos em idosos com um nível leve de comprometimento cognitivo.

Porém, os resultados acima apresentados não são consensuais, visto que conclusões opostas podem ser encontradas na literatura sobre o tema. Por exemplo, no estudo de Hänninen et al. (1994) as queixas relativas à perda de memória não se correlacionaram com o desempenho de participantes em testes. Porém, sujeitos que apresentaram mais queixas demonstraram também maior tendência de as verbalizar somáticas, bem como sentimentos de ansiedade relativos à sua condição de saúde.

Resultados similares foram apresentados por Derouesné, Lacomblez, Thibault e LePoncin (1999), que utilizaram um questionário de oito itens para avaliar a percepção de participantes em tarefas diárias que requeressem o uso da memória. Nesse estudo, quando comparados a pessoas da mesma faixa etária sem quaisquer queixas, idosos apre- 
sentam mais sentimentos negativos a respeito de suas capacidades. Em conjunto, os dois estudos recém mencionados sugerem que a apreciação subjetiva acerca da capacidade mnemônica esteja associada com sintomas depressivos e de ansiedade, não constituindo, dessa forma, um indicador da real performance dos sujeitos.

Embora seja reconhecida a importância da metamemória para autoavaliação sobre o funcionamento cognitivo, a queixa subjetiva, apesar de constituir uma medida promissora, ainda não está estabelecida como um indicador confiável de comprometimento cognitivo da memória (Clement, Belleville, \& Gauthier, 2008; Jungwirth et al., 2004). A divergência entre as pesquisas coloca em xeque a validade ecológica dos resultados e, principalmente, a interpretação dos mesmos. Em vista disso, este estudo aborda essa questão ainda controversa na literatura a partir da proposta de examinar se a percepção subjetiva de memória, mensurada a partir de um julgamento subjetivo, correlaciona-se com o desempenho obtido em testes objetivos de memória.

Optou-se por utilizar uma medida simples e direta (uma pergunta) para a avaliação das crenças dos participantes acerca de sua memória, à semelhança do estudo de Mattos et al. (2003). Em vista da observação de que ansiedade e depressão podem responder pelas avaliações negativas de idosos acerca de sua memória (Derouesné et al., 1999; Hänninen et al., 1994), foram coletadas medidas para esses fatores, tendo sido as mesmas utilizadas no cálculo das correlações parciais.

Ainda, dada a multiplicidade de domínios funcionais da memória humana, neste estudo foram utilizados testes específicos a esses domínios, de modo a tornar possível uma análise mais refinada dos resultados. De fato, conforme assinalam Sunderland, Harris e Baddeley (1983), a validade ecológica dos resultados em testes de memória tem relação direta com a qualidade e com a especificidade das medidas. A partir desse procedimento, como se verá, relações entre a percepção subjetiva e o desempenho em testes objetivos são encontradas em alguns domínios da memória.

\section{Método}

\section{Participantes}

A amostra foi composta por 152 idosos (138 mulheres) com idades entre 60 e 89 anos. A seleção dos participantes foi realizada por conveniência. Os idosos foram recrutados em grupos de convivência de Porto Alegre (RS). Todos os participantes eram autônomos, socialmente ativos, residentes na comunidade e frequentavam esses grupos para realizar atividades cognitivas, físicas e sociais. Como critérios de inclusão, os indivíduos deveriam ter 60 anos ou mais, ter pontuação inferior a cinco na Geriatric Depression Scale (GDS-15, Escala de Depressão Geriátrica) e inferior a 20 no Beck Anxiety Inventory (BAl, Inventário de Ansiedade de Beck), bem como apresentar pontuação igual ou superior a 18 no Mini-Exame do Estado Mental (MEEM), no caso de idosos com baixa/média escolaridade, ou igual ou superior a 26, nos de idosos com alta escolaridade (Bertolucci, Brucki, Campacci, \& Juliano, 1994).

\section{Instrumentos}

\section{Mini-Exame do Estado Mental}

Os instrumentos utilizados foram os seguintes:

1. Ficha de Dados Sociodemográficos: a partir desse instrumento foram coletados dados relativos ao sexo, à idade, à escolaridade e à renda dos participantes. Além disso, o instrumento investigou a percepção subjetiva de memória por meio de uma questão que avaliava como o idoso a percebia, oferecendo quatro opções de resposta: ótima, boa, regular e ruim.

2. Escala de Depressão Geriátrica-15: é uma medida utilizada para identificação e quantificação de sintomas depressivos em idosos. A versão curta é composta por 15 perguntas em relação à escala original (que apresenta 30), com respostas classificadas em "sim" ou "não". O escore total da GDS-15 é feito a partir do somatório das respostas 
assinaladas pelos examinandos nos 15 itens. 0 menor escore possível é zero, e o maior é 15 , sendo os escores entre 0 e 4 interpretados como normais. Escores entre 5 e 9 representam indício de depressão leve, e os situados entre 10 e 15 indicam a presença de depressão severa.

3. Inventário Beck de Ansiedade: trata-se de uma medida de nível de ansiedade composta por 21 itens. O escore total é feito a partir do somatório das respostas assinaladas pelos participantes em todos os itens. O maior possível é 63. Escores entre 0 e 7 representam níveis mínimos e normais de ansiedade; entre 8 e 15, presença de ansiedade leve; entre 16 e 25, ansiedade moderada; e a faixa de 26 a 63 indica níveis severos de ansiedade.

4. Mini-Exame do Estado Mental (Bertolucci et al., 1994): é um instrumento de avaliação de funções cognitivas composto por questões que avaliam orientação temporal e espacial, registro de palavras, atenção e cálculo, lembrança de palavras, linguagem e capacidade construtiva visual. O escore pode variar de zero até 30 pontos. Em sujeitos normais, são esperados escores superiores a 25; abaixo disso, há indício de perda da capacidade cognitiva, que pode ser leve (21-24 pontos), moderada (10-20) ou grave (escores inferiores ou iguais a 9). Apesar de o MEEM ter sido utilizado para os critérios de inclusão na amostra, a questão de número cinco foi utilizada nas comparações estatísticas, uma vez que avalia a memória dos participantes para objetos estudados. A pontuação máxima da questão é de 3 pontos.

5. Subtestes do Instrumento de Avaliação Neuropsicológica Breve (Neupsilin) (Pawlowski, Fonseca, Salles, Parente, \& Bandeira, 2008): esse instrumento possui tempo reduzido de aplicação (30-40 minutos). As 32 tarefas avaliam nove funções cognitivas: orientação têmporo-espacial; atenção; percepção; memória; habilidades aritméticas; linguagem; praxias; resolução de problemas; e função executiva de fluência verbal. Neste estudo foram utilizadas apenas as tarefas de memória, compostas pelos seguintes subtestes:

5.1 Memória de trabalho - ordenamento 154 ascendente de dígitos: repetição em ordem cres- cente de conjuntos de dois a seis dígitos. Pontuação: 0-10.

5.2 Memória de trabalho - span auditivo de palavras em sentenças: memorização e lembrança de palavras e frases pronunciadas pelo examinador. Pontuação: 0-28.

5.3 Memória episódica verbal - evocação imediata: lembrança de nove palavras emitidas pelo examinador. Pontuação máxima: 0-9.

5.4 Memória episódica verbal - evocação tardia: emissão das palavras do subteste anterior em tempo posterior. Pontuação máxima: 0-9.

5.5 Memória episódica verbal - reconhecimento: identificação das palavras anteriormente estudadas em uma lista de palavras maior. Pontuação: 0-18.

5.6 Memória semântica de longo prazo: resposta a duas perguntas relativas a conhecimentos gerais. Pontuação: 0-5.

5.7 Memória visual de curto prazo: memorização de uma figura sem sentido e posterior reconhecimento em um teste com três figuras. Pontuação: 0-3.

5.8 Memória prospectiva: lembrança da instrução de que se escreva o nome em uma folha de papel, fornecida no início da testagem. Pontuação: 0-2.

\section{Procedimentos}

Após realizado contato com os grupos de idosos, procedeu-se à inclusão dos participantes na amostra e à coleta de dados. Foi realizada uma entrevista individual, na qual cada idoso preencheu o Termo de Consentimento Livre e Esclarecido e respondeu à Ficha de Dados Sociodemográficos. Posteriormente, foram avaliados os sintomas depressivos (GDS-15) e as funções cognitivas através do MEEM e do Neupsilin.

O pacote estatístico Statistical Package for the Social Sciences (SPSS) (versão 20) foi utilizado para analisar os dados. A descrição das variáveis foi realizada por meio das frequências absolutas e relativas, bem como através da média e do desvio 
padrão. Foram verificados importantes desvios da normalidade a partir de níveis significativos no teste de Kolmogorov-Smirnov, bem como ausência de homogeneidade na variância em diversas variáveis. Por essa razão, as comparações entre os grupos utilizaram a estatística $U$ de Mann-Whitney.

Embora se tenha abandonado os pressupostos de normalidade em favor do uso de estatísticas não-paramétricas, o que sugere o uso do coeficiente de Spearman $\left(r_{s}\right)$ para o cálculo das correlações, o caráter dicotômico da variável "percepção de memória", após sua recodificação, permite o uso do coeficiente de Pearson ( $r$ ). Ainda, em vista da interação prevista entre a "percepção de memória" e a escolaridade dos participantes, bem como com os escores na GDS-15 e no BAI, esses fatores foram controlados por meio do cálculo de correlações parciais.

Por fim, considerando o caráter dicotômico da variável "percepção de memória" e, especialmente, em vista do pressuposto teórico de continuidade dos valores entre os níveis estabelecidos dessa variável, foi calculada a correlação bisserial para os índices $r$ de Pearson inicialmente significativos (tratados como correlações bisseriais por ponto). A correlação foi ajustada pela equação

$$
r_{b}=\frac{r_{p b} \sqrt{\left(\mathrm{P}_{1} \mathrm{P}_{2}\right)}}{\mathrm{y}}
$$

onde: $r_{b}$ é a correlação bisserial; $r_{p b}$ é correlação bisserial por pontos; $P 1$ e $P 2$ são as frequências da distribuição nos grupos $(0,513$ e 0,487 , respectivamente); e y é a ordenada da distribuição normal padrão (onde existe P1\% da área em um dos lados

Tabela 1

Características sociodemográficas, sintomas depressivos e de ansiedade em idosos. Porto Alegre (RS), 2009

\begin{tabular}{lcc}
\hline Indicador & Média & Desvio-Padrão \\
\hline Idade (anos) & 68,95 & 7,030 \\
Escolaridade (anos) & 12,82 & 4,234 \\
Renda (salários) & 3,39 & 1,620 \\
Escore total na escala GDS-15 & 1,96 & 2,210 \\
Escore total no BAl & 6,82 & 6,604 \\
\hline
\end{tabular}

Nota: BAl: Beck Anxiety Inventory; GDS-15: Geriatric Depression Scale-15. do eixo que passa por $Z=0$, e $P 2 \%$ do outro). Os níveis de significância dos sucessivos testes $U$ foram ajustados pela correção de Bonferroni para $\alpha=0,0055556$. Além disso, o projeto foi examinado e aprovado pelo Comitê de Ética em Pesquisa da Pontifícia Universidade Católica do Rio Grande do Sul, sob o n $07 / 03730$.

\section{Resultados}

A Tabela 1 apresenta os dados sociodemográficos da amostra em estudo. A pontuação média no GDS-15 (Média - $M=1,96$, Desvio-Padrão $D P=2,21)$ corresponde à ausência de sintomas depressivos, enquanto que o escore médio de 6,82 $(D P=6,60)$ no BAl sugere a presença de níveis mínimos e normais de ansiedade.

A observação da distribuição de respostas à pergunta "o que você acha de sua memória?" referente à "percepção subjetiva de memória" dos participantes evidenciou que: 14 idosos avaliaram sua memória como "ótima" (9,2\%); 64 avaliaram-na como "boa" (42,1\%); 58 como "regular" (38,2\%); e 16 como "ruim" (10,5\%). A Tabela 2 apresenta as médias e os desvios-padrão para os respectivos testes, de acordo com as quatro condições de percepção subjetiva de memória.

Para as demais análises estatísticas, recodificou-se a variável "percepção subjetiva de memória", transformando-a em dicotômica; as categorias "ótima" e "boa" em uma nova categoria denominada "boa"; e os níveis "regular" e "ruim" foram recodificados para uma categoria denominada como "ruim", referindo-se a uma percepção negativa acerca da própria memória. As novas frequências da distribuição, a partir da variável transformada, evidenciaram que 78 idosos avaliaram sua memória como "boa" (51,3\%), enquanto 74 avaliaram-na como "ruim" (48,7\%).

As comparações entre os grupos para os subtestes de memória são apresentadas na Tabela 3. A partir da estatística de Mann-Whitney, foram verificadas diferenças significativas quanto à percepção subjetiva apenas nos subtestes de memória de trabalho do Neupsilin (Ordenamento ascendente 
Tabela 2

Médias de pontuação nos Subtestes de Memória em idosos. Porto Alegre (RS), 2009

\begin{tabular}{|c|c|c|c|c|c|c|c|c|}
\hline \multirow{3}{*}{ Tarefa } & \multicolumn{8}{|c|}{ "O que você acha de sua memória?" } \\
\hline & \multicolumn{2}{|c|}{ Ótima $(n=14)$} & \multicolumn{2}{|c|}{ Boa $(n=64)$} & \multicolumn{2}{|c|}{ Regular $(n=58)$} & \multicolumn{2}{|c|}{ Ruim $(n=16)$} \\
\hline & $M$ & $D P$ & $M$ & $D P$ & $M$ & $D P$ & $M$ & $D P$ \\
\hline Lembranças de palavras (MEEM) & 2,29 & 0,47 & 2,48 & 0,59 & 2,34 & 0,71 & 2,06 & 1,00 \\
\hline Ordenamento ascendente de dígitos & 8,07 & 1,86 & 7,77 & 1,42 & 7,19 & 1,26 & 7,25 & 1,39 \\
\hline Span auditivo de palavras em sentenças & 16,79 & 3,74 & 16,53 & 4,26 & 14,74 & 4,19 & 12,94 & 4,04 \\
\hline Evocação imediata & 4,86 & 1,40 & 4,67 & 1,51 & 4,72 & 1,24 & 3,19 & 1,22 \\
\hline Evocação tardia & 0,93 & 1,38 & 1,95 & 1,99 & 1,95 & 1,83 & 0,81 & 1,05 \\
\hline Reconhecimento & 12,29 & 2,09 & 12,53 & 2,34 & 12,26 & 2,64 & 10,44 & 3,48 \\
\hline Memória semântica - longo prazo & 5,00 & 0,00 & 5,00 & 0,00 & 4,93 & 0,26 & 4,94 & 0,25 \\
\hline Memória visual - curto prazo & 2,57 & 0,51 & 2,50 & 0,64 & 2,57 & 0,60 & 2,50 & 0,52 \\
\hline Memória prospectiva & 1,29 & 0,47 & 1,36 & 0,70 & 1,26 & 0,71 & 0,94 & 0,77 \\
\hline
\end{tabular}

Nota: DP: Desvio-Padrão; M: Média; MEEM: Mini-Exame do Estado Mental.

Tabela 3

Comparações entre os grupos de idosos em relação à Percepção Subjetiva de Memória no MEEM e aos Subtestes de Memória do Neupsilin. Porto Alegre (RS), 2009

\begin{tabular}{|c|c|c|c|c|}
\hline \multirow{2}{*}{ Tarefa } & \multicolumn{2}{|c|}{ "O que você acha de sua memória?"a } & \multirow{2}{*}{$U^{\mathbf{b}}$} & \multirow{2}{*}{$p$} \\
\hline & Boa & Ruim & & \\
\hline Lembranças de palavras (MEEM) & 2,00 & 2,00 & 2643,000 & 0,320 \\
\hline Memória de trabalho - ordenamento ascendente de dígitos & 8,00 & 7,00 & 2089,500 & $0,003^{*}$ \\
\hline Memória de trabalho - span auditivo de palavras em sentenças & 16,50 & 14,00 & 2064,000 & $0,002^{*}$ \\
\hline Memória verbal - evocação imediata & 5,00 & 4,00 & 2499,500 & 0,145 \\
\hline Memória verbal - evocação tardia & 1,00 & 1,00 & 2868,500 & 0,947 \\
\hline Memória verbal - reconhecimento & 12,00 & 12,00 & 2570,500 & 0,241 \\
\hline Memória semântica - longo prazo & 5,00 & 5,00 & 2691,000 & 0,020 \\
\hline Memória visual - curto prazo & 3,00 & 3,00 & 2809,500 & 0,745 \\
\hline Memória prospectiva & 1,00 & 1,00 & 2566,000 & 0,198 \\
\hline
\end{tabular}

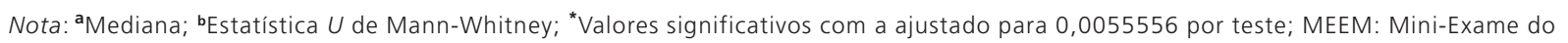
Estado Mental.

de dígitos, $p=0,003$; e Span auditivo de palavras em sentenças, $p=0,002$ ). Ou seja, ao menos no que se refere à memória de trabalho, os idosos que tinham uma percepção subjetiva positiva obtiveram melhor desempenho nas tarefas.

Por fim, a Tabela 4 apresenta as correlações entre o nível de escolaridade dos participantes e as tarefas de memória, bem como entre estas e os escores do BAl e do GDS-15. Uma vez que a relação entre esses fatores é conhecida, e considerando a significância estatística entre algumas correlações e as tarefas de memória, eles foram controlados no cálculo das correlações parciais entre os subtestes e a percepção de memória.
A análise correlacional (Tabela 4) também evidencia correlações parciais fracas, mas significativas, nas tarefas de "Memória de trabalho - Span auditivo de palavras em sentenças" ( $r=0,24$, $p=0,021)$ e "Memória semântica de longo prazo" $(r=0,27, p=0,007)$. Para o subteste "Memória de trabalho - ordenamento ascendente de dígitos", observou-se uma correlação fraca $(r=0,20$, $p=0,058)$ e marginalmente significativa.

\section{Discussão}

A validade da obtenção de medidas subjetivas de avaliação da memória tem sido abordada 
Correlações entre os Subtestes de Memória e os escores do Inventário Beck de Ansiedade, da Escala de Depressão Geriátrica e da Percepção Subjetiva em idosos. Porto Alegre (RS), 2009

\begin{tabular}{lccccc}
\hline Tarefa & Escolaridade $\left(r_{s}\right)$ & BA $\left(r_{s}\right)$ & GDS-15 $\left(r_{s}\right)$ & Percepção Subjetiva $(r)^{\mathbf{a}}$ & $\begin{array}{c}\text { Percepção Subjetiva } \\
\text { ajustada }\left(r_{b}\right)^{\mathbf{b}}\end{array}$ \\
\hline Lembranças de palavras (MEEM) & 0,052 & $-0,061$ & $-0,139$ & 0,096 & - \\
Ordenamento ascendente de dígitos & 0,131 & $-0,114$ & $-0,187^{*}$ & 0,156 & 0,20 \\
Span auditivo & $0,227^{* *}$ & $-0,116$ & $-0,136$ & $0,189^{*}$ & 0,24 \\
Evocação imediata & $0,217^{* *}$ & $-0,035$ & $-0,193^{*}$ & 0,058 & - \\
Evocação tardia & $0,185^{*}$ & 0,048 & $-0,095$ & 0,041 & - \\
Reconhecimento & 0,050 & $-0,051$ & $-0,118$ & 0,064 & - \\
Memória semântica - longo prazo & 0,022 & 0,012 & $-0,020$ & $0,218^{* *}$ & 0,27 \\
Memória visual - curto prazo & 0,091 & $-0,067$ & $-0,076$ & 0,081 & - \\
Memória prospectiva & 0,146 & 0,022 & $-0,123$ & 0,074 & - \\
\hline
\end{tabular}

Nota: ${ }^{a}$ Correlações parciais, controladas para o nível de Escolaridade e para os escores do BAl e da GDS-15; ${ }^{\mathbf{b}}$ Apenas as correlações significativas foram ajustas para correlações bisseriais; "Valores significativos em $p<0,05 ;{ }^{* *}$ Valores significativos em $p<0,01$.

BAl: Beck Anxiety Inventory; GDS-15: Geriatric Depression Scale-15; MEEM: Mini-Exame de Estado Mental.

em diversos estudos ao longo das últimas décadas. Tem-se sugerido, a exemplo do estudo de Cook e Marsiske (2006), a existência de correlações positivas entre escores objetivos em testes e avaliações subjetivas sobre o desempenho. Porém, a ausência de consenso quanto aos resultados denota a necessidade de se aprimorar as pesquisas, seja em seu desenho, seja em seus instrumentos e amostras.

Por um lado, observa-se uma variedade de ferramentas na mensuração da percepção individual dos idosos sobre sua memória, desde perguntas simples (e.g., Mattos et al., 2003) até múltiplos testes específicos (e.g., Cook \& Marsiske, 2006). Por outro lado, a lacuna no estabelecimento de uma relação próxima entre a percepção subjetiva de memória e a real performance de participantes em tarefas objetivas pode ser explicada por uma baixa validade ecológica dos testes usuais de memória (Sunderland et al., 1983). O estudo de Derouesné et al. (1999), por exemplo, apesar de demonstrar a ligação estreita entre medidas subjetivas versus objetivas, salienta a forte dependência entre as medidas utilizadas, visto que as correlações não são observadas quando se utiliza uma análise multivariada.

Por outro lado, considerando quadros clínicos mais severos, os achados de Geerlings, Jonker, Bouter, Adèr e Schmand (1999) sugerem que queixas de memória são preditoras da incidência de doença de Alzheimer em idosos nos quais o declínio cognitivo não é ainda aparente. Além disso, esses autores sugerem que pessoas idosas podem estar conscientes acerca do declínio das funções cognitivas quando testes mentais são, ainda, incapazes de detectar um declínio em direção ao funcionamento pré-mórbido.

No presente estudo, utilizou-se uma pergunta inserida em um questionário sociodemográfico de modo a avaliar a percepção de idosos acerca de sua memória. Medidas objetivas foram obtidas a partir de testes neuropsicológicos e, posteriormente, foram comparadas entre os grupos com avaliação boa ou ruim de sua memória. Além disso, correlações parciais foram obtidas entre os testes e a medida subjetiva (percepção de memória), controladas para os fatores de ansiedade, depressão e escolaridade, visto serem conhecidas as interações entre essas variáveis e a metamemória.

Os resultados obtidos indicam que a percepção subjetiva de memória, a qual pode ser representada clinicamente como uma queixa subjetiva vinculada à mesma, correlaciona-se fraca, mas significativamente, com o desempenho de idosos em testes de memória de trabalho (Span auditivo e no ordenamento ascendente de dígitos), sendo marginalmente significativa a correlação com o subteste relacionado à "Memória semântica de longo prazo". 
Dessa forma, os resultados indicam que a percepção subjetiva de memória está relacionada com um melhor desempenho de idosos em tarefas de memória de trabalho e de memória semântica de longo prazo. No sentido oposto, a percepção negativa está relacionada a um pior desempenho, e esse resultado é salutar quando se avalia a utilidade clínica dessa medida de metamemória. Porém, quando são consideradas as comparações entre os grupos com avaliação boa ou ruim, valores significativos são obtidos apenas nos subtestes de memória de trabalho, apoiando parcialmente os resultados das correlações.

Portanto, os dados apresentados neste estudo representam evidências sobre a validade da avaliação subjetiva de memória em sua relação com o desempenho de idosos em testes clínicos. O fato de que as diferenças foram manifestas apenas nas tarefas relacionadas à memória de trabalho sugere futuras investigações que avaliem a interação da percepção individual com a especificidade de domínios funcionais da memória. Porém, é necessário tomar tais resultados de forma cautelosa. Embora sejam significativos, as correlações são fracas e, consequentemente, é baixa a variância explicada.

Uma limitação importante deste estudo foi a forma de obtenção do julgamento subjetivo, restrito a uma questão simples acerca da crença dos participantes em quão boa era sua memória. Não obstante o reconhecimento desse aspecto, deve-se notar que Mattos et al. (2003) utilizaram uma abordagem equivalente. De fato, esses autores argumentam que o questionamento direto é uma maneira eficaz de se abordar a questão.

Sugere-se que estudos posteriores na população brasileira façam uso de abordagens mais abrangentes quanto à obtenção das medidas subjetivas, e mais específicas em relação às objetivas. Uma possível solução é o uso de instrumentos específicos para a avaliação da percepção que sujeitos têm acerca de seu próprio desempenho e da qualidade de sua memória, ou mesmo daqueles que abordem diretamente as queixas subjetivas de memória. A adaptação de instrumentos como o Cognitive Failures Questionnaire (Broadbent,
Cooper, FitzGerald, \& Parkes, 1982) e o Illness

Perception Questionnaire - Memory (Hurt, Burns, Brown, \& Barrowclough, 2010) pode auxiliar na obtenção de dados mais precisos.

\section{Colaboradores}

F.R. BOURSCHEID e L. MOTHES participaram na concepção e desenho do estudo, análise de dados e redação final. T.Q. IRIGARAY participou na concepção e desenho do estudo, coleta de dados e revisão do artigo.

\section{Referências}

Bertolucci, P. H., Brucki, S. M., Campacci, S. R., \& Juliano, Y. (1994). O mini-exame do estado mental em uma população geral: impacto da escolaridade. Arquivos de Neuropsiquiatria, 52(1), 1-7. http://dx.doi.org/10. 1590/S0004-282X1994000100001

Broadbent, D. E., Cooper, P. F., FitzGerald, P., \& Parkes, K. R. (1982). The Cognitive Failures Questionnaire (CFQ) and its correlates. British Journal of Clinical Psychology, 21, 1-16.

Chaves, M. L. F., Godinho, C. C., Porto, C. S., Mansur, L., Carthery-Goulart, M. T., Yassuda, M. S., \& Beato, R. (2011). Cognitive, functional and behavioural assessment: Alzheimer's disease. Dementia and Neuropsychologia, 5(3), 153-166.

Clement F., Belleville S., \& Gauthier S. (2008). Cognitive complaint in mild cognitive impairment and Alzheimer's Disease. Journal of the International Neuropsychological Society, 14, 222-232. http://dx. doi.org/10.1017/S1355617708080260

Cook, S., \& Marsiske, M. (2006). Subjective memory beliefs and cognitive performance in normal and mildly impaired older adults. Aging \& Mental Health, 10(4), 413-423. http://dx.doi.org/10.1080/136078606006 38487

Derouesné, C., Lacomblez, L., Thibault, S., \& LePoncin, M. (1999). Memory complaints in young and elderly subjects. International Journal of Geriatric Psychiatry, 14, 291-301. http://dx.doi.org/10.1002/(SICI)10991166(199904)14:4<291::AID-GPS902>3.0.CO;2-7

Dixon, R. A., Hultsch, D. F., \& Hertzog, C. (1988). The Metamemory in Adulthood (MIA) Questionnaire. Psychopharmacology Bulletin, 24(4), 671-688.

Dufouil, C., Fuhrer, R., \& Alpérovitch, A. (2005). Subjective cognitive complaints and cognitive decline: Consequence or predictor? The epidemiology of vascular aging study. Journal of the American Geriatrics Society, 53(4), 616-621.

Geerlings, M. I., Jonker, C., Bouter, L. M., Adèr, H. J., \& Schmand, B. (1999). Association between memory 
complaints and incident Alzheimer's disease in elderly people with normal baseline cognition. American Journal of Psychiatry, 156(4), 531-537.

Gilewski, M. J., Zelinski, E. M., \& Schaie, K. W. (1990). The memory functioning questionnaire for assessment of memory complaints in adulthood and old age. Psychology and Aging, 5, 482-490. http://dx.doi.org/ 10.1037/0882-7974.5.4.482

Hänninen, T., Reinikainen, K. J., Helkala, E. L., Koivisto, K., Mykkänen, L., Laakso, M., \& Riekkinen, P. J. (1994). Subjective memory complaints and personality traits in normal elderly subjects. Journal of the American Geriatrics Association, 42(1), 1-4.

Hurt, C. S., Burns, A., Brown, R. G., \& Barrowclough, C. (2010). Perceptions of subjective memory complaint in older adults: The Illness Perception Questionnaire Memory (IPQ-M). International Psychogeriatrics, 22(5), 750-760. http://dx.doi.org/10.1017/S104161020999 1542

Jonker, C., Launer, L. J., Hooijer, C., \& Lindeboom, J. (1996). Memory complaints and memory impairment in older individuals. Journal of the American Geriatrics Society, 44, 44-49.

Jonker, C., Geerlings M. I., \& Schmand, B. (2000). Are memory complaints predictive for dementia? A review of clinical and population-based studies. International Journal of Geriatric Psychiatry, 15(11), 983-991.
Jungwirth, S., Fischer, P., Weissgram, S., Kirchmeyr, W., Bauer, P., \& Tragl, K. H. (2004). Subjective memory complaints and objective memory impairment in the Vienna-Transdanube aging community. Journal of the American Geriatrics Society, 52, 263-268. http://dx. doi.org/10.1111/j.1532-5415.2004.52066

Mattos, P., Lino, V., Rizo, L., Alfano, A., Araújo, C., \& Raggio, R. (2003). Queixas de memória de idosos saudáveis e desempenho em testes. Arquivos de Neuropsiquiatria, 61(4), 920-924. http://dx.doi.org/10. 1590/S0004-282X2003000600006

Pawlowski, J., Fonseca, R. P., Salles, J. F., Parente, M. A. M. P., \& Bandeira, D. (2008). Evidências de validade do instrumento de avaliação neuropsicológica breve NEUPSILIN. Arquivos Brasileiros de Psicologia, 60, 101-116.

Souchay, C. (2007). Metamemory in Alzheimer's disease. Cortex, 43, 987-1003. http://dx.doi.org/10.1016/S001 0-9452(08)70696-8

Sunderland, A., Harris, J. E., \& Baddeley, A. (1983). Do laboratory tests predict everyday memory: A neuropsychological study. Journal of Verbal Learning and Verbal Behavior, 22(3), 341-357. http://dx.doi.org/ 10.1016/s0022-5371(83)90229-3

Recebido: maio 10, 2013

Versão final: maio 13, 2014

Aprovado: setembro 22, 2014 
